\title{
Off-Line Learning and the Primary Motor Cortex
}

\author{
Edwin M. Robertson, ${ }^{1}$ Daniel Z. Press, ${ }^{1}$ and Alvaro Pascual-Leone $e^{1,2}$ \\ ${ }^{1}$ Center for Non-Invasive Brain Stimulation, Beth Israel Deaconess Medical Center, Boston, Massachusetts 02215, and ${ }^{2}$ Institut Guttmann, Hospital de \\ Neurorehabilitació, Universitat Autonoma de Barcelona, 08916 Barcelona, Spain
}

\begin{abstract}
We are all familiar with acquiring skills during practice, but skill can also continue to develop between practice sessions. These "off-line" improvements are frequently supported by sleep, but they can be time dependent when a skill is acquired unintentionally. The magnitude of these over-day and overnight improvements is similar, suggesting that a similar mechanism may support both types of off-line improvements. However, here we show that disruption of the primary motor cortex with repetitive transcranial magnetic stimulation blocks off-line improvements over the day but not overnight. This suggests that a memory may be rescued overnight and subsequently enhanced or that different aspects of a skill, with differential dependencies on the primary motor cortex, are enhanced over day and overnight. Off-line improvements of similar magnitude are not supported by similar mechanisms; instead, the mechanisms engaged may depend on brain state.
\end{abstract}

Key words: motor cortex; sensorimotor; motor learning; motor control; learning and memory; repetitive transcranial magnetic stimulation

\section{Introduction}

Motor skills are acquired during practice, and they can also continue to develop between practice sessions. These "off-line" improvements can develop over wake and sleep. A similar magnitude of improvement develops across these intervals when a skill is acquired unintentionally in a procedural sequence-learning task (Robertson et al., 2004a). This suggests that a similar neural circuit may support off-line learning across these time intervals. Alternatively, changes in brain state across the interval may determine the circuit of brain areas that support off-line improvements.

The primary motor cortex (M1) is one area within a circuit of brain areas that has been implicated in the acquisition of motor skills during practice (Seidler et al., 2002). After practice, the M1 continues to make an important contribution to procedural learning by playing a critical role in the stabilization of procedural memories (Muellbacher et al., 2002). This stabilization is a form of procedural consolidation, which unlike off-line learning, does not produce a between-session skill improvement but instead makes a skill less susceptible to interference (Robertson et al., 2004b; Walker, 2005). Whether the M1 also makes a critical contribution to off-line learning and whether intervening changes in brain state modify this contribution remain unknown.

Using transcranial magnetic stimulation (TMS) to disrupt the function of the M1, we explored the contribution of the M1 to

Received Jan. 18, 2005; revised May 26, 2005; accepted May 27, 2005.

This work was supported by the National Alliance for Research in Schizophrenia and Depression (E.M.R.), the National Institutes of Health (MH-65434 to D.Z.P., RR018875 to A.P.-L.), and the Goldberg Foundation (A.P.-L). We are grateful to Melynda Casement and Gregory Clement for recruiting participants to this study.

Correspondence should be addressed to Edwin M. Robertson, Center for Non-Invasive Brain Stimulation, Beth Israel Deaconess Medical Center, 330 Brookline Avenue, Kirstein Building KS 454, Boston, MA 02215. E-mail: emrobert@bidmc.harvard.edu.

DOI:10.1523/JNEUROSCI.1851-05.2005

Copyright $\odot 2005$ Society for Neuroscience $\quad$ 0270-6474/05/256372-07\$15.00/0 off-line learning with (8 P.M. to 8 A.M.) and without (8 A.M. to 8 P.M.) intervening sleep. Participants acquired skill in performing a sequence of finger movements in the serial reaction time task (SRTT) (Nissen and Bullemer, 1987). This task was introduced to participants as a test of reaction time. When described in this way, skill is acquired unintentionally, and there are off-line improvements over day and overnight (Robertson et al., 2004a).

After skill acquisition either in the morning (8 A.M.) or the evening (8 P.M.), TMS was applied over the M1. By stimulating after skill acquisition, we ensured that only off-line processing, and not the initial encoding of the new skill, was affected by TMS (see Fig. 1). Skill was retested $12 \mathrm{~h}$ later. Performance during retesting was unlikely to have been influenced by any residual effect of TMS because these dissipate within $\sim 10$ min (Iyer et al., 2003; Robertson et al., 2003). With two types of $12 \mathrm{~h}$ interval (over day, 8 A.M. to 8 P.M.; overnight, 8 P.M. to 8 A.M.) and two types of stimulation (sham and real), there were four groups of participants: (1) overnight/TMS, (2) overnight/sham, (3) day/ TMS, and (4) day/sham. To ensure that the effects of TMS on off-line learning over the day were temporally and spatially specific, we stimulated over the M1 $2 \mathrm{~h}$ (day/TMS $+2 \mathrm{~h}$ ) after the first session, and in another group, we stimulated $25 \mathrm{~mm}$ anterior to the M1 (day/TMS $+25 \mathrm{~mm}$ ). In a final group, skill was retested 15 min after applying TMS to the M1 at either 8 A.M. or 8 P.M. This allowed the immediate effects of TMS on a procedural memory to be assessed.

\section{Materials and Methods}

Participants. Fifty individuals with no neurological or psychiatric conditions were recruited to this study. All were naive to the purpose of this institutional review board-approved study, all gave informed consent for the study, and all met the additional safety criteria for repetitive TMS (rTMS) (Wassermann, 1998). At the end of the study, six participants were removed from additional analysis because they were able to recall, in a free recall test, more than four items of the 12-item sequence of finger 
movements. This greater-than-chance recall suggests that these participants had become aware of acquiring a new skill (Goedert and Willingham, 2002), which can prevent the development of off-line improvements over the day (Robertson et al., 2004a). Thirty-six participants (20.8 \pm 2.5 years of age; 19 male) were distributed randomly and equally among six off-line groups (see Fig. 1). The remaining eight participants (19.9 \pm 1.4 years of age; five male) were distributed equally between two groups. These additional groups were designed to assess the immediate effect of TMS on a procedural memory.

Experimental design. Performance in a sequence learning task (see below) was measured before and after a $12 \mathrm{~h}$ interval (see Fig. $1 \mathrm{~A}$ ). This interval either did (8 P.M. to 8 A.M.) or did not (8 A.M. to 8 P.M.) include sleep. After participants had learned the sequence of finger movements (see Fig. 1A, session 1), TMS or sham stimulation was immediately applied to the M1. Previous studies have suggested that a procedural memory is probably most susceptible to the effects of TMS immediately after its encoding (Muellbacher et al., 2002; Baraduc et al., 2004). With two interval types (sleep or no sleep) and two types of stimulation (TMS or sham), there were four groups: (1) overnight/TMS, (2) overnight/sham, (3) day/TMS, and (4) day/sham. All overnight participants kept a sleep log. There were two additional day groups. Immediately after sequence learning, TMS was applied $25 \mathrm{~mm}$ anterior to the M1 (day/TMS $+25 \mathrm{~mm}$ ); and in a final group, TMS was applied to the M1, $2 \mathrm{~h}$ after (day/TMS $+2 \mathrm{~h}$ ) participants had learned the finger-movement sequence (see Fig. $1 B$ ). These groups were designed to demonstrate that the effects of TMS were spatially and temporally specific. In two final groups, skill was retested $15 \mathrm{~min}$ after TMS had been applied to the M1 at either 8 A.M. or 8 P.M., allowing the immediate effect of TMS on skill to be accessed. This design allowed the effects of TMS on time-dependent skill improvements to be examined without affecting the initial encoding or later retrieval of the finger movement sequence (see Fig. $1 B$ ). The initial acquisition of the finger-movement sequence was not affected by TMS because stimulation was applied after training. At retesting, the primary neurophysiological effects of stimulation will have dissipated (Chen et al., 1997; Iyer et al., 2003; Robertson et al., 2003).

Procedural-sequence learning. A modified version of the SSRT (Nissen and Bullemer, 1987) was used to guide the acquisition of a fingermovement sequence. A solid blue circular stimulus (diameter, $20 \mathrm{~mm}$; viewed from $\sim 800 \mathrm{~mm}$ ) appeared on a monitor at one of four possible positions within an equally spaced horizontal array. The presentation of each stimulus was controlled by a computer using software designed specifically to record response times (Superlab; Cedrus, San Pedro, CA). Each of the four possible positions corresponded to one of the four buttons on a response pad (RB-410; Cedrus), on which the participants' fingers rested. When a target appeared, participants were instructed to respond by pressing the appropriate button on the pad as quickly and as accurately as possible. Participants responded using the fingers of their preferred hand [Edinburgh questionnaire (Oldfield, 1971)]. Having made the correct response, the cue on the screen disappeared and was replaced by the next cue after a delay of $400 \mathrm{~ms}$. If the participant made an incorrect response, the stimulus remained until the correct button was selected. The task was described to participants as a test of reaction time; however, the position of each cue followed a regular and repeating 12item pattern (2-3-1-4-3-2-4-1-3-4-2-1).

Participants unintentionally learned this sequence of finger movements during the first session and were retested on the same sequence during the second session (see Fig. 1A). The first session consisted of three blocks: a training block sandwiched between two test blocks. Later, during the second session, participants performed a single test block (see Fig. $1 \mathrm{~A}$ ). The training block had 25 repetitions ( 300 trials) of the 12 -item sequence. The two test blocks had only 15 repetitions (180 trials). These were designed to probe participants' skill before $\left(\right.$ Skill $\left._{1}\right)$ and after $\left(\right.$ Skill $\left._{2}\right)$ the $12 \mathrm{~h}$ interval, giving them little opportunity to acquire additional skill during either test block (Robertson et al., 2004a). When participants are tested and then later retested 15 min later on the test blocks, there is no significant increase in their skill (Robertson et al., 2004a). These blocks are, therefore, not sufficient to support skill acquisition; instead, they probe skill before and after the time interval between sessions.

Fifty random trials preceded and followed the sequential trials in the training and test blocks (see Fig. $1 A$ ). Within these random trials, there were no item repeats, and each item had approximately the same frequency of appearance. Each of the groups used the same set of random trials.

After retesting, a participant's awareness for the underlying sequence was assessed using a free-recall test. Awareness was defined as correctly recalling more than four consecutive items of the sequence (Willingham and Goedert-Eschmann, 1999). All participants who recalled five or more items were removed from subsequent analysis.

Transcranial magnetic stimulation. Stimulation was delivered using a Magstim (Whitland, Wales, UK) Super Rapid stimulator. The position of the M1 was defined by finding the location where the lowest intensity of stimulation was capable of inducing visible abductor pollicus brevis muscle contractions in at least 6 of 10 trials (Wassermann et al., 1996). This intensity of stimulation defined the motor threshold (MT). We applied a $1 \mathrm{~Hz}$ train of rTMS for $10 \mathrm{~min}$ (600 pulses) at $90 \%$ of MT over the M1 or $25 \mathrm{~mm}$ anterior to the M1. These stimulation parameters are known to alter cortical excitability and behavior (Robertson et al., 2003). The figure of eight coil (commercially available double $70 \mathrm{~mm}$ coil; Magstim) was positioned tangentially to the scalp with the handle of the coil $135^{\circ}$ from the midsagittal axis of the participant's head and the coil pointing posterior (Mills et al., 1992). For sham stimulation, we used a commercially available sham coil that looks identical to the coil used for real stimulation (double $70 \mathrm{~mm}$ sham coil; Magstim). The center of this sham coil was placed $50 \mathrm{~mm}$ lateral to the $\mathrm{Cz}$ electrode position (vertex) from the international 10-20 system, a site that approximately overlies the M1. Sham stimulation was delivered at $1 \mathrm{~Hz}$ for $10 \mathrm{~min}$ (600 pulses); this was associated with auditory stimulation similar to that induced by real stimulation and some cutaneous sensation. Participants naive to TMS, as all participants were, are unable to differentiate between this form of sham TMS and real TMS.

Data analysis. Only the response times for correct responses were included in the analysis. Any response time longer than 2.7 SDs (i.e., the top $1 \%$ ) from a participant's mean was removed, as was any response time exceeding $3000 \mathrm{~ms}$. A learning score was calculated by subtracting the average response time of the final 50 random trials from the average response time of the preceding 50 sequential trials. This widely used measure of skill factors out nonspecific influences on response times to give a specific measure of procedural sequence learning (Nissen and Bullemer, 1987; Willingham et al., 1989; Willingham and GoedertEschmann, 1999). Accuracy in the SRTT is not a useful measure of skill, because even with limited experience, error rates are extremely low $(<1-$ $2 \%)$. Skill before the interval ( Skill $_{1}$ ) was calculated using the final test block of the first session, and skill after the interval $\left(\mathrm{Skill}_{2}\right)$ was calculated using the first and only test block of the second session (see Fig. 1A). The difference $\left(\right.$ Skill $_{2}-$ Skill $\left._{1}\right)$ between these learning scores gave a measure of off-line learning. A Kruskal-Wallis test was used to compare the amount of skill and off-line learning across the experimental groups, and a Mann-Whitney $U$ test used to make planned comparisons between groups. To test for off-line learning within each group, a Wilcoxon signed ranks test was used to compare Skill ${ }_{1}$ against Skill 2 . These nonparametric tests were used because some groups had a skill and an offline learning distribution that differed significantly (at the 5\% level) from a Gaussian distribution, according to a Lilliefors test. Across the six groups, a repeated-measures ANOVA was used to explore the possibility that sequential response times, which did follow a Gaussian distribution, changed within the retest block. This block was divided into three equal sets of 60 trials. An average response time was calculated for each of these sets, giving a measure of change in response time across the retest block, for each participant in each group. We also used an ANOVA to seek for differences among the groups in participants' MT and free recall of the sequence.

\section{Results}

\section{The contribution of the M1 to off-line learning}

Before the interval, there was no significant difference in skill (Fig. 1, Skill 1 ) across the groups (Kruskal-Wallis test; $\chi^{2}=7.709$; $\mathrm{df}=5 ; p=0.173$ ) (Fig. 2). Twelve hours later, skill was retested (Fig. 1, Skill ${ }_{2}$ ), and a between session change in skill (Skill ${ }_{2}-$ 
A

B

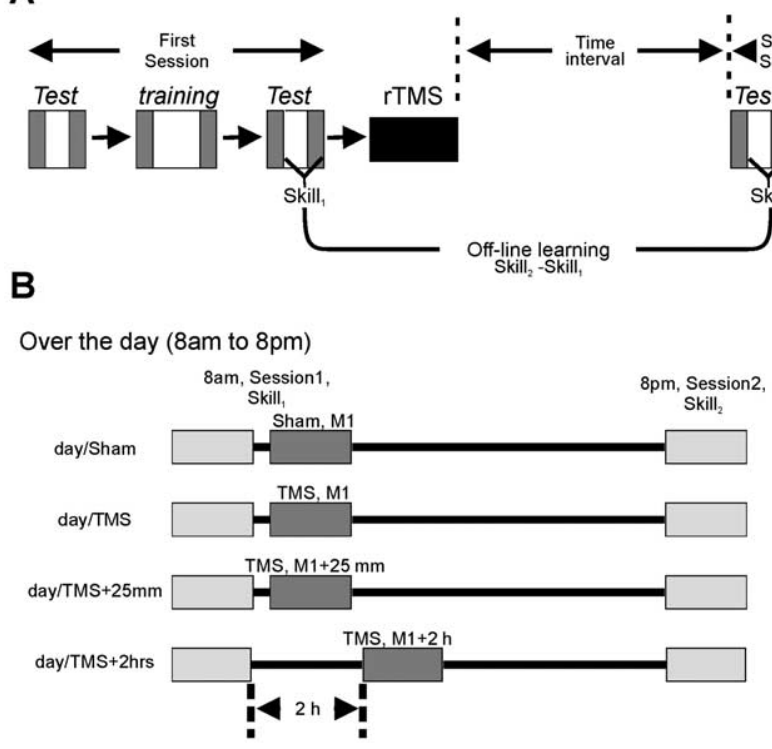

Overnight (8pm to $8 \mathrm{am})$

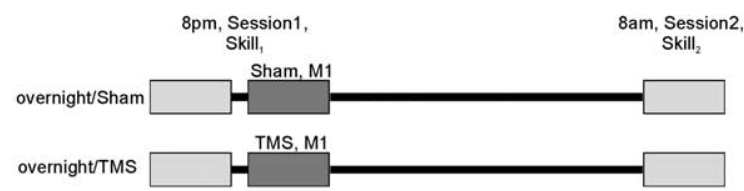

Figure 1. Experimental design. $A$, The first session consisted of a training block sandwiched between two test blocks. The final session consisted of only a single test block. $\boldsymbol{B}$, Real or sham TMS (dark gray box) was applied after initial skill learning (Skill ${ }_{1}$; Session 1; light gray box) and at least $10 \mathrm{~h}$ before skill was retested (Skill ${ }_{2}$; session 2; light gray box). This prevented TMS from affecting the initial encoding (session 1) of the skill or its later retrieval (session 2). The skill difference before $\left(S k i l_{1}\right)$ and after $\left(\right.$ Skill $\left._{2}\right)$ the interval gave the amount of off-line learning (Skill ${ }_{2}-$ Skill $_{1}$ ). We examined the effect of stimulation (real or sham) on off-line learning over the day (8 A.M. to 8 P.M.) or overnight (8 P.M. to 8 A.M.). The temporal and spatially specificity of TMS was examined by stimulating over the $\mathrm{M} 12 \mathrm{~h}$ (day/TMS $+2 \mathrm{~h}$ ) after the first session, and in another group, we stimulated $25 \mathrm{~mm}$ anterior to $\mathrm{M1}$ (day/TMS $+25 \mathrm{~mm}$ ).

Skill ${ }_{1}$ ) was calculated. The effect of stimulation on these off-line changes differed depending on whether the interval extended over the day or overnight (Kruskal-Wallis test; $\chi^{2}=14.047 ; \mathrm{df}=$ $3 ; p=0.003$ ) (Fig. 2). Over-the-day improvements were substantially greater after sham (day/sham) than after real (day/TMS) stimulation to the M1 (Mann-Whitney $U$ test; $Z=2.882 ; p=$ 0.004) (Figs. 2, 3). After sham stimulation to the M1, there were substantial improvements (Wilcoxon signed ranks test; $Z=$ 2.201; $p=0.028$ ), but after real stimulation, there was a slight but nonsignificant decrease in skill (Wilcoxon signed ranks test; $Z=$ 1.782; $p=0.075$ ) (Fig. 3). In contrast, both overnight groups showed significant off-line learning (Wilcoxon signed ranks test; $Z=3.059 ; p=0.002$ ). Overnight improvements after real compared with sham stimulation were not significantly different (Mann-Whitney $U$ test $Z=1.441 ; p=0.18$ ). The improvements either over the day or overnight after sham stimulation did not differ significantly (Mann-Whitney $U$ test $Z=0.320 ; p=0.749$ ). After initial testing, participants in the two overnight groups spent an additional $3.6 \mathrm{~h}( \pm 0.2 \mathrm{~h}$; sleep logs) awake before retiring to bed. This interval did not differ significantly between the two overnight groups (unpaired $t$ test; $t_{(10)}=0.450 ; p=0.662$ ). These participants then reported sleeping for an average of $6.7 \mathrm{~h}$ $( \pm 0.3 \mathrm{~h}$; sleep logs). Both groups reported sleeping for approximately the same length of time (unpaired $t$ test; $t_{(10)}=1.17 ; p=$
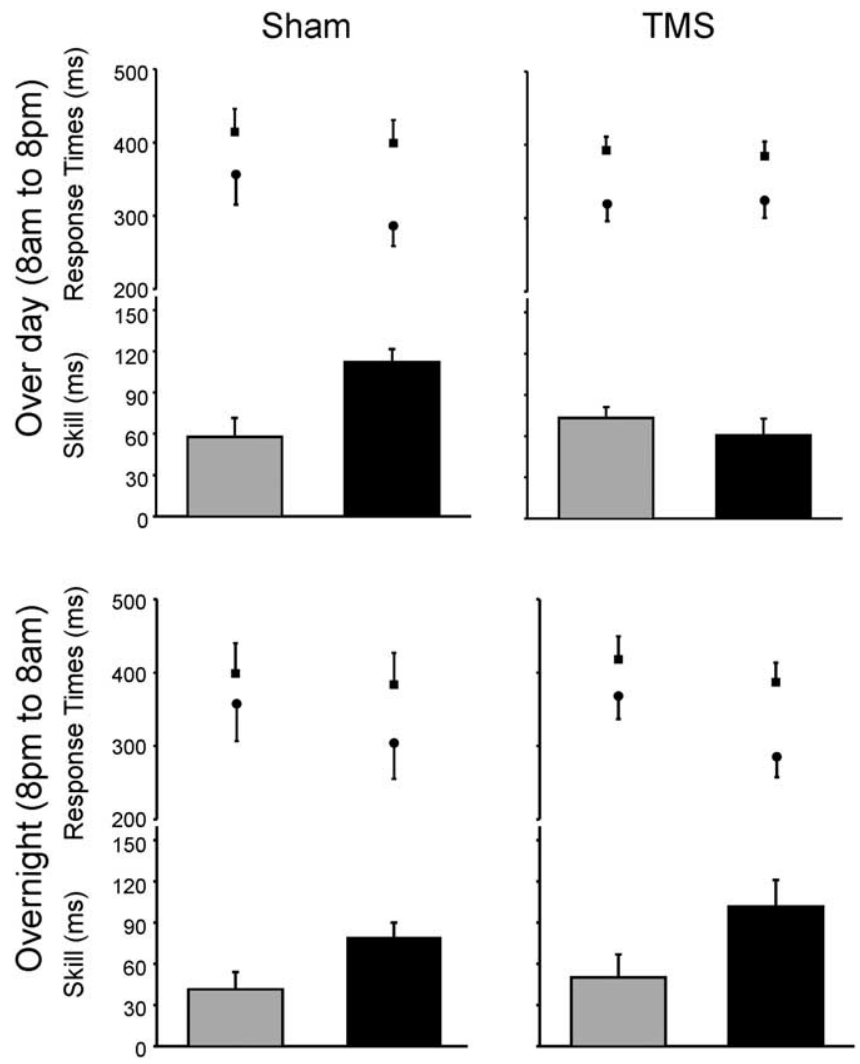

Figure 2. Off-line improvements over the day (8 A.M. to 8 P.M.) and overnight (8 P.M. to 8 A.M.) after sham or real TMS. At initial testing, skill (Skill $;$; gray boxes) did not differ significantly among the four groups (Kruskal-Wallis test; $\chi^{2}=6.1 ; \mathrm{df}=3 ; p=0.12$ ). At retesting, skill had significantly increased (Skill 2 ; black boxes) in all but one of the groups; TMS applied to the M1 blocked over-the-day improvements. In contrast, overnight improvements were not affected by stimulation; improvements after sham or real stimulation did not differ significantly (Mann-Whitney $U$ test; $Z=1.441 ; p=0.18$ ). The difference between response times to random (square \pm SE) and sequential trials (circle \pm SE) trials is a standard measure of skill for this sequence learning task (Nissen and Bullemer, 1987). The random response times did not differ significantly across the groups (ANOVA; $F_{(3,20)}=0.079 ; p=0.97$ ).

0.269). Participants in the over day groups reported spending the period awake performing daytime activities (e.g., studying, eating, and exercising).

These observations show that TMS applied to the M1 is able to block off-line learning over the day but not overnight. Therefore, different mechanisms, differentially involving the M1, are engaged to support over-the-day and overnight off-line learning.

\section{Spatially and temporally specific effect of TMS}

The ability of TMS to block off-line learning over the day was both spatially and temporally specific. When the TMS coil was positioned $25 \mathrm{~mm}$ anterior to the M1 (day/TMS $+25 \mathrm{~mm}$ ), there were significant improvements over the $12 \mathrm{~h}$ interval (Wilcoxon signed ranks test; $Z=2.201 ; p=0.028$ ) (Fig. 3). These increases were significantly greater than the skill changes observed when the coil was positioned directly over the M1 (Mann-Whitney $U$ test; $Z=2.722 ; p=0.006$ ). Similarly, there were significant improvements when the application of TMS over the M1 was delayed by $2 \mathrm{~h}$ (day/TMS $+2 \mathrm{~h}$; Wilcoxon signed ranks test; $Z=$ 2.201; $p=0.028$ ) (Fig. 3), and these improvements were greater than when TMS was applied immediately after the initial acquisition of the finger movement sequence (Mann-Whitney $U$ test; $Z=2.882 ; p=0.004)$. Skill improvements did not differ significantly across this delay group (day/TMS $+2 \mathrm{~h}$ ), the group that 


\section{A Over the day (8am to $8 \mathrm{pm}$ )}

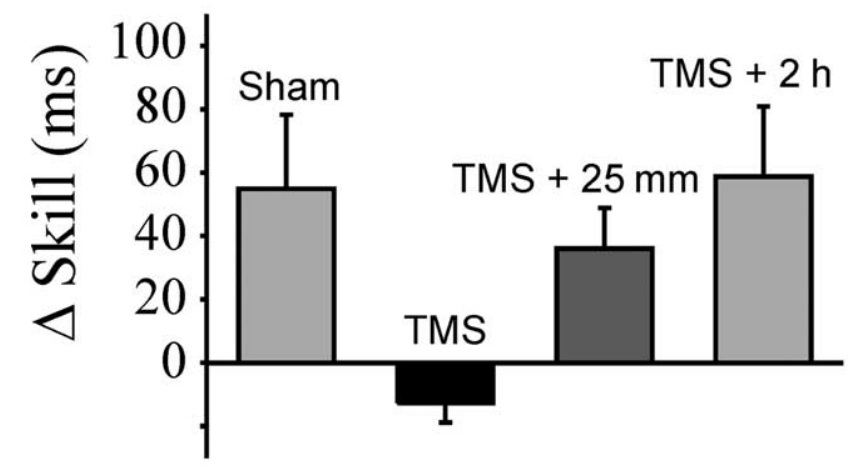

\section{B Overnight (8pm to 8am)}

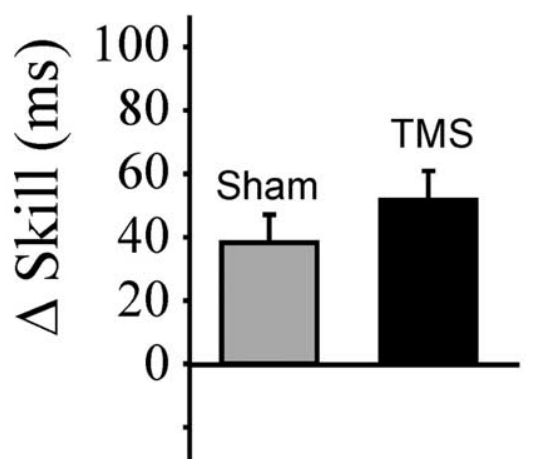

Figure 3. $\quad \boldsymbol{A}$, Over-the-day, off-line improvements were blocked when TMS was applied to the M1 (TMS). This was both a spatially and temporally specific affect; off-line improvements were not blocked when TMS was applied $25 \mathrm{~mm}$ anterior to the M1 (TMS $+25 \mathrm{~mm}$ ) or $2 \mathrm{~h}$ after skill acquisition (TMS $+2 \mathrm{~h}$ ). $\boldsymbol{B}$, In contrast, applying TMS to the M1 could not block overnight improvements. After sham stimulation, the amount of off-line learning over the day or overnight did not differ significantly (Mann-Whitney $U$ test; $Z=0.320 ; p=0.749$ ). Error bars represent SEM.

received TMS $25 \mathrm{~mm}$ anterior to the $\mathrm{M} 1$ (day/TMS $+25 \mathrm{~mm}$ ), or those that received sham stimulation (day/sham) to the M1 (Kruskal-Wallis test; $\chi^{2}=0.924 ; \mathrm{df}=2 ; p=0.630$ ). Finally, there was no decrease in sequential response time across the retest block in any of the six groups (ANOVA; $F_{(10,60)}=0.80 ; p=$ 0.625) (Fig. 4).

\section{Sequence recall}

Off-line learning can occur over the day and overnight, provided participants recall less than five items of the sequence (Robertson et al., 2004a). Scoring above this guessing rate led to the removal of six participants from the study (Willingham and GoedertEschmann, 1999). Only data from the remaining participants is described. On average, these participants recalled 0.7 items in a free-recall test, a score that did not vary significantly across the groups $\left(\right.$ ANOVA; $\left.F_{(5,30)}=0.802 ; p=0.557\right)$. Only four participants, only one of whom was in the day/TMS group, recalled even four items of the sequence; most did not recall even a single item.

\section{Cortical excitability}

The ability of TMS to specifically block over-day, but not overnight, improvements, cannot be attributed to changes in the sus-

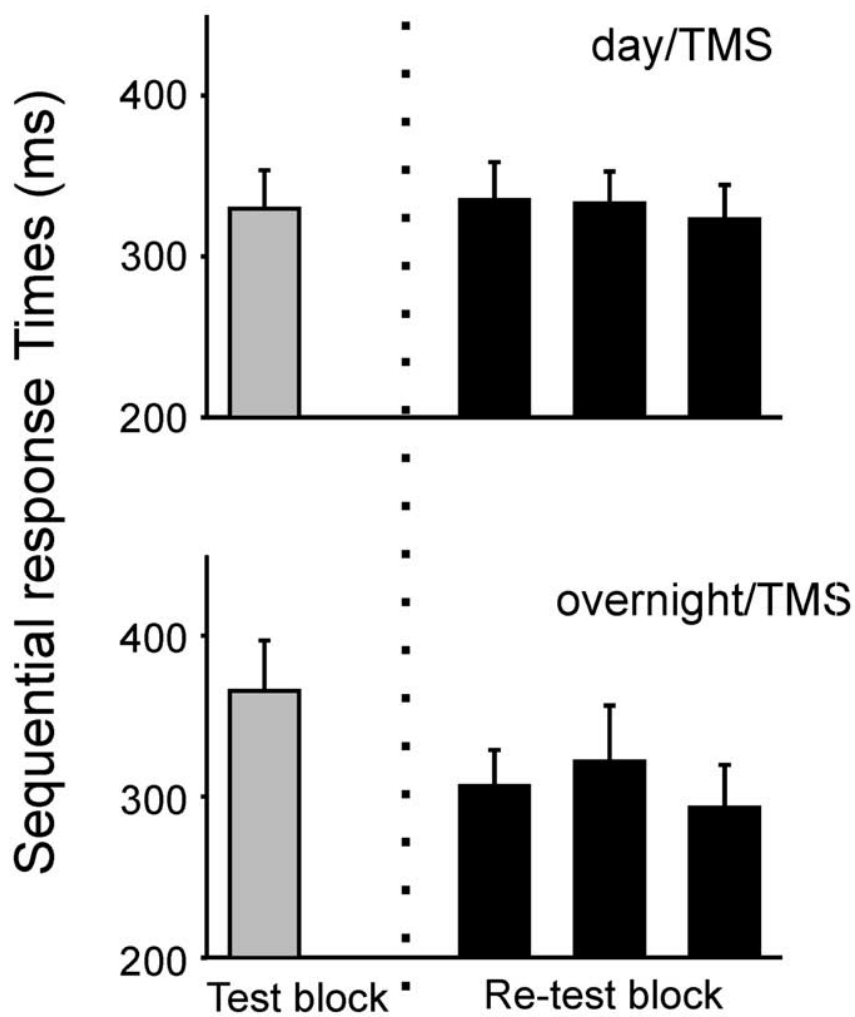

Figure 4. Response times of the final 60 sequential response times of the test block (gray bar) and the 180 trials of the retest block divided into three sets (black bars) of 60 sequential response times. There was no significant change in response time across these three sets in either the day/TMS (ANOVA; $\left.F_{(2,4)}=0.76 ; p=0.525\right)$ or the overnight/TMS (ANOVA; $F_{(2,4)}=$ $1.64 ; p=0.301)$ group. This lack of change in response time was observed across the six groups (ANOVA; $F_{(10,60)}=0.80 ; p=0.625$ ). Consequently, as was suggested in a previous study, the retest block was not sufficient to support the acquisition of skill (Robertson et al., 2004a). Instead, the improvement in sequential response time develops between the test and retest block. There was a significant fall in sequential response time between the final 60 trials of the test block and the first 60 trials of the retest block in the overnight/TMS group (paired $t$ test; $t_{(5)}=3.378 ; p=0.02$ ) but no significant fall in the day/TMS group (paired $t$ test; $t_{(5)}=0.349$; $p=0.741$ ). There was no difference between the last 60 sequential response times (test block; session 1; gray bar) of the day/TMS and the overnight/TMS group (unpaired $t$ test; $t_{(10)}=0.912$; $p=0.383$ ). These observations are consistent with off-line improvements being blocked in the day/TMS group but not in the overnight/TMS group. Error bars represent SEM.

ceptibility of the M1 to stimulation at different times of day. Motor threshold, a measure of cortical excitability and susceptibility of the M1 to stimulation (Robertson et al., 2003), did not vary significantly across the groups (ANOVA; $F_{(3,20)}=0.347 ; p=$ $0.792)$. MT, and several other measures of cortical excitability, also show little diurnal variation (Manganotti et al., 2001; Strutton et al., 2003).

\section{Immediate effects of TMS}

Before the application of TMS to the M1, there was no significant difference between the skill acquired at 8 A.M. and 8 P.M. (MannWhitney $U$ test; $Z=0.289 ; p=0.773$ ). Fifteen minutes after stimulation, skill was retested. There was no significant change in skill between testing and retesting at either 8 A.M. (Wilcoxon signed ranks test; $Z=1.095 ; p=0.273$ ) or 8 P.M. (Wilcoxon signed ranks test; $Z=0.730 ; p=0.465$ ). The small skill changes observed at 8 A.M. and 8 P.M. were not significantly different (Mann-Whitney $U$ test; $Z=1.433 ; p=0.2$ ). There was still no evidence of a skill change, between testing and retesting, even 
when the two groups were combined (Wilcoxon signed ranks test; $Z=0.28 ; p=0.779)$. At retesting, skill was significantly greater than a naive (i.e., a $0 \mathrm{~ms}$ response time difference between sequential and random trials) performance (Wilcoxon signed ranks test; $Z=2.521 ; p=0.012$ ). In this and two previous studies, 15 min lapsed between stimulation of the M1 and retesting (Muellbacher et al., 2002; Baraduc et al., 2004). This should allow sufficient time for the neurophysiological effects of $10 \mathrm{~min}$ of 1 Hz TMS to mostly dissipate (Chen et al., 1997; Robertson et al., 2003), while not giving sufficient time for off-line improvements to develop (Robertson et al., 2004a; Press et al., 2005). None of these eight participants were able to recall more than four items of the sequence, and participants' recall did not differ significantly between the two groups (Mann-Whitney $U$ test; $Z=$ 0.683; $p=0.495$ ), nor was there a significant difference in MT when it was recorded at 8 A.M. or 8 P.M. (Mann-Whitney $U$ test; $Z<0.1 ; p=0.99$ ).

\section{Discussion}

TMS applied to the M1 after practice blocks off-line learning over the day. Thus, during the day, a neural circuit that includes the M1 makes a critical contribution to off-line learning. In contrast, when there is an interval of sleep, improvements are not dependent on M1 integrity; TMS applied to the M1 was unable to prevent substantial overnight improvements. Although improvements develop overnight and over the day, the neural circuits supporting these improvements appear to differ. Consistent with these observations are recent functional imaging studies showing that the M1 is not engaged during overnight improvements (Maquet et al., 2000, 2003). However, the brain areas engaged during off-line learning over the day have not been directly examined, nor has any study contrasted the neural circuits supporting off-line learning over wake and overnight.

\section{The spatial and temporal influence of TMS}

The impairment of over day improvements, but not overnight improvements, was a specific effect of TMS. Sham stimulation showed no evidence of preventing over-day or overnight skill improvements. During the day, improvements were only blocked when TMS was applied over the M1 immediately after participants had learned a finger-movement sequence. When stimulation was delayed by $2 \mathrm{~h}$ or applied $25 \mathrm{~mm}$ anterior to M1, improvements still developed. This demonstrates that the effects of TMS on off-line learning were both temporally and spatially specific. A previous study achieved a similar spatial specificity when TMS was used to block the stabilization, as opposed to the offline improvement, of skills. In accord with previous behavioral studies (Brashers-Krug et al., 1996), it showed that at least $6 \mathrm{~h}$ is required for the stabilization of skills (Brashers-Krug et al., 1996; Muellbacher et al., 2002). In contrast, the off-line development of skill appears to receive critical support form a network that includes the M1 for at most $2 \mathrm{~h}$. Improvements may take at least $4 \mathrm{~h}$ to develop; consequently, only the first couple of hours of this process appears to be critically dependent on a network that includes the M1 (Press et al., 2005).

The effects of stimulation are unlikely to be restricted to the M1. Combined functional imaging and TMS studies have demonstrated that a distributed circuit of brain areas is activated by stimulation (Paus, 1999). Applying $1 \mathrm{~Hz}$ TMS for 15 min to the M1 activates a circuit of brain areas (Siebner et al., 2003). Even the effects of $10 \mathrm{~min}$ of stimulation are unlikely to be restricted to the M1. Consequently, when TMS is applied to the M1, a circuit of brain areas, including the M1, is disrupted. This circuit appears to make a critical contribution to the development of off-line improvements over the day but not overnight.

\section{Possible contamination from skill acquired during practice}

Our results cannot be explained by skill being acquired during retesting. Skill learning during the retest block would be expected to cause a decrease in the response time of the sequential trials. However, there was no significant change in sequential response time across the retest block in any group (Fig. 4). Furthermore, there was also no skill increase with an interval of only $15 \mathrm{~min}$ between testing and retesting sessions (Robertson et al., 2004a). An improvement, therefore, is not an obligatory consequence of performing the retest block.

\section{Participants' recall of the sequence}

A participant's awareness of the sequence cannot explain our results. Improvements occur over the day provided participants have little awareness of the sequence (Robertson et al., 2004a). In this study, any participant who recalled more than four items of the 12-item sequence was removed from additional analysis. Consequently, all participants had a rate of recall that was substantially beneath the rate of recall (eight items) associated with exclusively overnight improvements (Robertson et al., 2004a). Furthermore, the rate of recall did not differ significantly across the groups.

\section{Memory stability and enhancement}

Typically, TMS exerts its influence on behavior by disrupting ongoing neurophysiological events; when the disruption dissipates, so do the behavior changes associated with stimulation (Robertson et al., 2003). However, by disrupting a memory, TMS is able to affect performance long after its primary neurophysiological affects have dissipated (Muellbacher et al., 2002; Baraduc et al., 2004). After skill acquisition, stimulation over the M1 can return performance to a naive level (Muellbacher et al., 2002). This implies that some procedural memories are fragile immediately after their encoding. This, however, is only true for some memories (Robertson et al., 2004b). For example, the sequence learning task used in this study shows little evidence of fragility after encoding (Goedert and Willingham, 2002). Consistent with this lack of fragility, stimulation did not return skill to a naive level; instead, there was no significant difference between skill at testing and retesting 15 min later. Similarly, skill at producing accurate finger movements in a force field remains unchanged between testing and retesting, despite stimulation of the M1 immediately after skill acquisition (Baraduc et al., 2004). In the current study, similar insignificant skill changes, between testing and retesting, were observed at both 8 A.M. and 8 P.M. Consequently, there is little evidence that TMS has a differential impact on the stability of a procedural memory when applied to the M1 at 8 A.M. or 8 P.M. Nonetheless, stimulation is able to block over the day but not overnight skill improvements.

\section{Sleep and TMS}

The possible effect of TMS on sleep, although an interesting and important question, cannot explain our observations, because similar overnight improvements developed after sham and real stimulation. Consequently, any effect that TMS may have had on sleep architecture did not impact on the overnight skill improvements. It appears unlikely that stimulation would have an effect on sleep architecture. A few studies specifically designed to examine this issue failed to find any substantial changes in sleep architecture after the application of TMS (Cohrs et al., 1998; Graf et al., 
2001). Furthermore, in our study, participants in the two overnight groups spent on average $3.6 \mathrm{~h}$ awake before sleep. This gave sufficient time for the primary neurophysiological effects of TMS to dissipate, making it very unlikely that TMS was able to influence the following night of sleep (Robertson et al., 2003).

The overnight groups did not examine the effects of sleep in isolation. On average, participants spent $6.7 \mathrm{~h}$ of the $12 \mathrm{~h}$ interval asleep. In contrast, members of the over-day groups reported spending the $12 \mathrm{~h}$ engaged in daytime activities. Stimulation over the M1 prevented off-line improvements in the later but not the former case. Determining whether the M1 made a critical contribution to off-line learning and whether this contribution was altered over day or overnight was the aim of this study. Our observations suggest that a network of brain areas including the M1 does make a critical contribution to off-line learning over the day but not over a night of sleep. The duration of overnight sleep and the onset of sleep relative to the time of stimulation may be important factors influencing this differential affect of stimulation. Examining these issues was not the purpose of this study, nor would it allow us to distinguish between two possible explanations for our observations.

\section{Two possible explanations}

Practice may not produce just a single representation. Instead, there maybe multiple representations, each coding for different aspects of a sequence (Verwey and Wright, 2004). For example, one representation may code for the movement, whereas another may represent the goal of the movement (Grafton et al., 1998). Applying TMS to the M1 may disrupt one of these representations, yet others may remain. These additional representations could be processed off-line to produce between-session improvements. These other representations may only be available for off-line processing overnight. Enhancing these representations may require chemical or physiological changes that only occur during sleep (Hobson and Pace-Schott, 2002), or these enhancements may be conditional on circadian factors that occur overnight.

Another possibility is the brain-state changes that occur overnight may allow the representation originally disrupted by TMS to be reformed. For example, during sleep, the corrupted memory may be reorganized, allowing relevant associations to be strengthened and the memory to be salvaged (Fenn et al., 2003). Once the representation has been "recovered," it would be available for off-line processing and so could support the development of overnight improvements.

Common to these explanations is that a procedural memory is disrupted by stimulation. Yet, the skill expressed before and immediately after stimulation is similar. A disrupted memory could take many forms. Here, perhaps, the disruption prevents the memory from being enhanced but does not alter the propensity to express skill.

These results provide insight into the organization of consolidation. Multiple processes are engaged during consolidation so that a memory can be stabilized or enhanced (Robertson et al., 2004b; Walker, 2005). Our results show that even skill enhancement is not a single process; instead, different mechanisms, the engagement of which are modified by brain-state changes over day and overnight, can support similar off-line improvements. One of these mechanisms, which operates over the day, is mediated by a network of brain areas including the M1. These results demonstrate that this network is not only critical for skill acquisition, during practice, but it is also critical to the development of skill when practice has ceased (Sanes and Donoghue, 2000). However, this network is only critical to off-line learning over the day, not over a night of sleep.

\section{References}

Baraduc P, Lang N, Rothwell J, Wolpert D (2004) Consolidation of dynamic motor learning is not disrupted by rTMS of primary motor cortex. Curr Biol 14:252-256.

Brashers-Krug T, Shadmehr R, Bizzi E (1996) Consolidation in human motor memory. Nature 382:252.

Chen R, Classen J, Gerloff C, Celnik P, Wassermann EM, Hallett M, Cohen LG (1997) Depression of motor cortex excitability by low-frequency transcranial magnetic stimulation. Neurology 48:1398-1403.

Cohrs S, Tergau F, Riech S, Kastner S, Paulus W, Ziemann U, Ruther E, Hajak G (1998) High-frequency repetitive transcranial magnetic stimulation delays rapid eye movement sleep. NeuroReport 9:3439-3443.

Fenn KM, Nusbaum HC, Margoliash D (2003) Consolidation during sleep of perceptual learning of spoken language. Nature 425:614-616.

Goedert K, Willingham D (2002) Patterns of interference in sequence learning and prism adaptation inconsistent with the consolidation hypothesis. Learn Mem 9:279-292.

GrafT, Engeler J, Achermann P, Mosimann UP, Noss R, Fisch HU, Schlaepfer TE (2001) High frequency repetitive transcranial magnetic stimulation (rTMS) of the left dorsolateral cortex: EEG topography during waking and subsequent sleep. Psychiatry Res 107:1-9.

Grafton ST, Hazeltine E, Ivry RB (1998) Abstract and effector-specific representations of motor sequences identified with PET. J Neurosci 18:9420-9428.

Hobson J, Pace-Schott E (2002) The cognitive neuroscience of sleep: neuronal systems, consciousness and learning. Nat Rev Neurosci 3:679-693.

Iyer MB, Schleper N, Wassermann EM (2003) Priming stimulation enhances the depressant effect of low-frequency repetitive transcranial magnetic stimulation. J Neurosci 23:10867-10872.

Manganotti P, Palermo A, Patuzzo S, Zanette G, Fiaschi A (2001) Decrease in motor cortical excitability in human subjects after sleep deprivation. Neurosci Lett 304:153-156.

Maquet P, Laureys S, Peigneux P, Fuchs S, Petiau C, Phillips C, Aerts J, Del Fiore G, Degueldre C, Meulemans T, Luxen A, Franck G, Van Der Linden M, Smith C, Cleeremans A (2000) Experience-dependent changes in cerebral activation during human REM sleep. Nat Neurosci 3:831-836.

Maquet P, Schwartz S, Passingham R, Frith C (2003) Sleep-related consolidation of a visuomotor skill: brain mechanisms as assessed by functional magnetic resonance imaging. J Neurosci 23:1432-1440.

Mills KR, Boniface SJ, Schubert M (1992) Magnetic brain stimulation with a double coil: the importance of coil orientation. Electroencephalogr Clin Neurophysiol 85:17-21.

Muellbacher W, Ziemann U, Wissel J, Dang N, Kofler M, Facchini S, Boroojerdi B, Poewe W, Hallett M (2002) Early consolidation in human primary motor cortex. Nature 415:640-644.

Nissen MJ, Bullemer P (1987) Attentional requirements of learning: evidence from performance measures. Cognit Psychol 19:1-32.

Oldfield R (1971) The assessment and analysis of handedness: the Edinburgh inventory. Neuropsychologia 9:97-113.

Paus T (1999) Imaging the brain before, during, and after transcranial magnetic stimulation. Neuropsychologia 37:219-224.

Press DZ, Casement M, Pascual-Leone A, Robertson EM (2005) The time course of off-line motor sequence learning. Brain Res Cogn Brain Res, in press.

Robertson EM, Theoret H, Pascual-Leone A (2003) Studies in cognition: the problems solved and created by transcranial magnetic stimulation. J Cogn Neurosci 15:948-960.

Robertson EM, Pascual-Leone A, Press DZ (2004a) Awareness modifies the skill-learning benefits of sleep. Curr Biol 14:208-212.

Robertson EM, Pascual-Leone A, Miall RC (2004b) Current concepts in procedural consolidation. Nat Rev Neurosci 5:576-582.

Sanes JN, Donoghue JP (2000) Plasticity and primary motor cortex. Annu Rev Neurosci 23:393-415. 
Seidler RD, Purushotham A, Kim SG, Ugurbil K, Willingham D, Ashe J (2002) Cerebellum activation associated with performance change but not motor learning. Science 296:2043-2046.

Siebner HR, Filipovic SR, Rowe JB, Cordivari C, Gerschlager W, Rothwell JC, Frackowiak RSJ, Bhatia KP (2003) Patients with focal arm dystonia have increased sensitivity to slow-frequency repetitive TMS of the dorsal premotor cortex. Brain 126:2710-2725.

Strutton P, Maria C, Davey N (2003) Stability of corticospinal excitability and grip force in intrinsic hand muscles in man over a 24 -hr period. Physiol Behav 79:679-682.

Verwey W, Wright D (2004) Effector-independent and effector dependent learning in the discrete sequence production task. Psychol Res 68:64-70.

Walker MP (2005) A refined model of sleep and the time course of memory formation. Behav Brain Sci 28:40-51.
Wassermann EM (1998) Risk and safety of repetitive transcranial magnetic stimulation: report and suggested guidelines from the International Workshop on the Safety of Repetitive Transcranial Magnetic Stimulation, June 5-7, 1996. Electroencephalogr Clin Neurophysiol 108:1-16.

Wassermann EM, Wang B, Zeffiro TA, Sadato N, Pascual-Leone A, Toro C, Hallett M (1996) Locating the motor cortex on the MRI with transcranial magnetic stimulation and PET. NeuroImage 3:1-9.

Willingham D, Goedert-Eschmann K (1999) The relation between implicit and explicit learning: evidence for parallel development. Psychol Sci 10:531-534.

Willingham DB, Nissen MJ, Bullemer P (1989) On the development of procedural knowledge. J Exp Psychol Learn Mem Cogn 15: $1047-1060$. 